\title{
Rheological behavior of hydrophobically modified hydroxyethyl cellulose solutions: A linear viscoelastic model
}

\author{
A. Maestro, ${ }^{\text {a) }}$ C. González, and J. M. Gutiérrez \\ Chemical Engineering and Material Science Department, Barcelona University, \\ c/Martí i Franquès 1, 08028 Barcelona, Spain
}

(Received 17 April 2001; final revision received 2 October 2001)

\begin{abstract}
Synopsis
The rheological behavior of hydrophobically modified hydroxyethyl cellulose (HMHEC), an associative thickener, was studied and compared with that of hydrophobically modified ethoxylated urethanes (HEURs) and nonassociative celluloses. In contrast to HEURs, a simple Maxwell model does not fit the linear viscoelastic behavior of HMHEC. Differences are attributed to the stiffness and comb structure of HMHEC. A generalized Maxwell model with a logarithmic distribution of relaxation times is proposed, and another parameter that includes Rouse-like relaxation is added to fit behavior at high frequencies. Four parameters are needed to describe HMHEC viscoelasticity: a mean relaxation time, $\lambda_{M}$; its corresponding standard deviation, $\sigma$; a plateau modulus, $G_{N}$; and a viscosity at infinite frequency, $\eta_{\infty}$. Satisfactory fitting is obtained for all concentrations and temperatures in the range of frequencies studied. The sharp increase of $G_{N}$ with concentration indicates loop-to-bridge transitions. Temperature does not influence $G_{N}$, since the reduction in the number density of elastically effective chains caused by Brownian motion masks the direct effect of temperature. The dependence of $\lambda_{M}$ on temperature follows the Arrhenius equation, as does the relaxation time of HEURs, but it does not change with concentration, presumably because the comb structure of HMHEC prevents the formation of long superchains. (c) 2002 The Society of Rheology. [DOI: $10.1122 / 1.1427910]$
\end{abstract}

\section{INTRODUCTION}

Environmental and safety considerations make water-borne coatings interesting substitutes for solvent-borne ones. These paints require the addition of thickeners in order to achieve the characteristic rheological behavior desired in their use. Complete rheological characterization of thickener water solutions would facilitate formulation of new paints, and must include viscoelastic characterization.

Thickeners traditionally used are soluble derivatives of cellulose, such as hydroxyethyl celluloses of high molecular weight (HEC) [Wetzel et al. (1996)]. These derivatives thicken the medium because of chain entanglements between the long polymer molecules. When a shear stress is applied to them, chains disentangle and align themselves along the flow field, and viscosity decreases. They thus present shear-thinning behavior. Coatings thickened with HEC have some limitations (e.g., poor leveling and spattering) and in the last few years have been substituted in some applications by associative polymers, which consist of a hydrophilic backbone, where hydrophobic groups have been

\footnotetext{
${ }^{a)}$ Author to whom correspondence should be addressed. Electronic mail: maestro@angel.qui.ub.es
} 
added so that each molecule has two or more hydrophobes. Since those hydrophobic groups have mutual affinity, they form aggregates that are similar to micelles present in surfactant solutions. Hence, these thickeners are termed associative polymers [Yekta et al. (1993)].

Hydrophobe modification of chemically different families is commonly used in paint thickeners. Three of these polymers are the most common: hydrophobically modified alkali swellable polymers, hydrophobically modified celluloses, and hydrophobically modified ethoxylated urethanes. This last type, commonly called HEURs, has been widely studied [Glass et al. (1991); Karunasena et al. (1989); Annable and Buscall (1993); Yekta et al. (1993); Kaczmarski and Glass (1993); Svanholm et al. (1997); Xu et al. (1996); Tam et al. (1998)]. Telechelic HEURs consist of a linear water-soluble poly(oxyethylene) backbone extended with diisocyanate groups and end-capped with alkyl groups [Jenkins et al. (1991)]. Hydrophobes are located at the ends of the polymer chain. The most interesting feature of these molecules is that their viscoelastic properties can be described by a single-element Maxwell model because the relaxation process is dominated by a single relaxation time. Experimental data are therefore easily fitted to equations and quantitative studies can be performed. Annable and Buscall (1993) developed the micellar network theory, a modification of the transient network theory first developed by Green and Tobolsky (1946) and later modified by Tanaka and Edwards (1992). This theory is now widely accepted and is based on the association of polymer end groups to form micelle-like junctions, demonstrated by fluorescent experiments [Wang and Winnik (1990); Yekta et al. (1995)]. At low polymer concentrations in water, both ends of each chain occupy the same micelle, and the hydrophilic backbone is stretched, forming a loop in such a way that independent flower micelles are formed [Semenov et al. (1995)]. At higher polymer concentrations there are more micelles in the medium, thus micelles are closer together and a secondary association process occurs, in which two hydrophobes of the same chain participate in adjacent micelles, a bridge being formed between them. If bridges are extended to neighboring micelles and then to the whole solution, a three-dimensional micellar network is formed, which increases viscosity and confers viscoelastic characteristics to the medium. Annable and co-workers (1994) identified the relaxation time $\lambda$ with the inverse of the exit rate of the hydrophobe from the micelle $\beta_{0}$, which has an Arrhenius dependence on temperature

$$
\beta_{0}=\lambda^{-1}=\omega_{0} e^{\left(-E_{m} / k T\right)},
$$

where $E_{m}$ is the activation energy for disengagement and $\omega_{0}$ is a characteristic frequency of thermal vibration. Like Yekta et al. (1993), Annable proposed loop-to-bridge transitions when concentration increases or shear-stress decreases, and bridge-to-loop transitions that produce breakdown of the structure with shear-thinning behavior when high shear stress is applied.

Although telechelic HEUR viscoelasticity has been extensively studied in a quantitative way, and a theory for the thickening mechanism has been developed, this is not the case for other associative thickeners, such as hydrophobically modified celluloses. Sau and Landoll (1989) and Goodwin et al. (1989) have argued that the mechanism of these polymers is qualitatively similar to that of HEURs. That is, the thickening is due to hydrophobic interactions [Landoll (1982); Brown (1994)]. But there is a remarkable difference between the two groups. Associative celluloses do not behave as simple Maxwell fluids, since rheological data cannot be fitted with only one relaxation time, as recent studies demonstrate [Svanholm et al. (1997); Karlson et al. (2000)]. Svanholm and coworkers emphasized that hydrophobically modified hydroxyethyl cellulose (HMHEC) does not behave as a Maxwell fluid, in contrast to telechelic HEURs. So, it seems difficult 
TABLE I. Thickeners used in experiments.

\begin{tabular}{lcrrr}
\hline \hline & Acronym & $M_{W}\left(\mathrm{~g} \mathrm{~mol}^{-1}\right)$ & DS & MS \\
\hline 2-hydroxyethyl cellulose & HEC9 & 90000 & 1.5 & 2.5 \\
2-hydroxyethyl cellulose & HEC25 & 250000 & 1.0 & 2.0 \\
2-hydroxyethyl cellulose & HEC72 & 720000 & 1.5 & 2.5 \\
2-hydroxyethyl cellulose & HEC130 & 1300000 & 1.5 & 2.5 \\
2-hydroxyethyl cellulose, hydrophobically & HMHEC & 560000 & 2.0 & $2.7-3.4$ \\
Modified with hexadecyl groups & & & \\
\hline \hline
\end{tabular}

to find a model for oscillating measurements that allows calculation of parameters interesting for the understanding of the behavior of these polymers (such as the high frequency storage modulus or the relaxation time). Svanholm attributed this behavior to the stiffness of the backbone. On the other hand, Xu et al. (1997) found that comb HEURs also required a distribution of relaxation times, although their backbone is flexible. Karlson and co-workers (2000) studied HMHEC and used as a characteristic time both the crossover of $G^{\prime}$ and $G^{\prime \prime}$ and the inverse of the angular frequency at which the complex viscosity $\eta^{*}$ changes from Newtonian to shear thinning. These two times were not the same, as they ought to be if the Maxwell model could be applied, and were therefore useful only for comparative purposes. Karlson and co-workers admitted that a range of relaxation times should be used to appropriately fit oscillatory results. Other works have been published that study interaction of HMHEC with some components present in a paint, such as latex [Kroon (1993)], or surfactants [Sivadasan and Somasundaran (1990); Tanaka et al. (1992); Piculell et al. (1996)]. However, like the others, they failed to use an acceptable model to describe rheology. In fact, alternative tools should be employed to study these solutions. For example, these last authors used the $G^{\prime}$ and $G^{\prime \prime}$ dependence on the surfactant concentration at an arbitrary frequency. But results were susceptible to change depending on the frequency chosen and therefore could be used only for comparison.

Of interest, then, is a model capable of describing the viscoelasticity of associative celluloses with only a few parameters that are independent of frequency and that have physical significance. The study of the evolution of these parameters with given variables, like temperature and concentration, will help to understand the thickening mechanism and the differences observed with HEURs. This article describes a model that fits oscillatory experimental data through a distribution of relaxation times. The requirement of this distribution will be justified comparing the architecture of the associative cellulose and HEURs. The dependence of the fitting parameters on temperature and concentration is also discussed and compared with the micellar network theory developed for HEURs.

\section{EXPERIMENT}

\section{A. Materials and preparation}

Hydroxyethyl celluloses of different molecular weights and hydrophobically modified hydroxyethyl cellulose (HMHEC) were obtained from Aldrich. Their molecular weight $M_{w}$, molar substitution (MS) and degree substitution (DS) are summarized in Table I.

The celluloses were received as a solid powder and were used as supplied. De-ionized water further purified through a Millipore MilliQ purification system was used to prepare solutions. Water was stirred and heated to $40^{\circ}$, and polymer was gradually added. Stirring was maintained for $2 \mathrm{~h}$ and solutions were stored for at least $24 \mathrm{~h}$ before use to assure 
complete hydration. Solutions were used only in the period of 7 days in order to avoid undesirable effects produced by enzymatic degradation.

\section{B. Test methods}

\section{Interfacial tension measurements}

Interfacial tension measurements between water solutions of HMHEC and toluene were performed at room temperature, using a KRÜSS K12 tensiometer, through the Wilhelmy Plate method.

\section{Capillary viscometry}

Viscosity of dilute solutions was determined with an Ubbelohde capillary viscometer supplied by SCHOTT-GERÄTE, with the number 53203/0c. It is provided with an automatic measurement system of the AVS/S ViscoClock series. Temperature was controlled at $25 \pm 0.2{ }^{\circ} \mathrm{C}$. A concentrated solution was prepared and subsequent dilutions started from this.

\section{Oscillatory measurements}

Oscillatory measurements were performed using a HAAKE RS100 controlled stress rheometer. Two different sensors were used, depending on the viscoelasticity range of the solutions. A double cone $60 \mathrm{~mm}$ in diameter and with a $1^{\circ}$ cone angle was used for solutions with less viscosity, and a cone-and-plate sensor $60 \mathrm{~mm}$ in diameter and with a $4^{\circ}$ cone angle was used for the others. Some measurements were carried out with both sensors, and results were compared to assure that experimental data were not influenced by them. Temperature was controlled with a deviation of less than $\pm 0.2^{\circ} \mathrm{C}$. Frequency sweep measurements were carried out in the frequency range of $0.001-10$ or $0.01-10$ Hz. Stress was chosen in order to work within the linear viscoelastic region. To ensure that these conditions were met, preliminary stress sweeps were made at different frequencies before each measurement to determine the linear range. Stress was selected to produce a strain of less than $20 \%$.

\section{RESULTS AND DISCUSSION}

\section{A. Interfacial tension measurements}

Interfacial tension measurements were made to establish that HMHEC really associates to form micelle-like aggregates, as other associative polymers do. If HMHEC thickening is thought to be due to hydrophobic associations and in the present work HMHEC rheology is compared with that of HEURs their behavior must be proved to be at least similar. Interfacial tensions between HMHEC water solutions and toluene were measured at different concentrations and room temperature. Results are plotted in Fig. 1.

The change in slope of the straight lines obtained implies that some kind of hydrophobic aggregation occurs in the parts per million ( $\mathrm{ppm}$ ) concentration range. The concentration at which this change is seen can be termed critical aggregation concentration (cac), on the analogy of critical micellar concentration of surfactants. It seems to be very low, much lower than that usually observed for conventional surfactants. Around the concentration where bend is observed, individual molecules of polymer start to associate to form micelle-like aggregates. As concentration is very low, these aggregates are expected to be independent and free to move all around the solution. In this way, they can be identified with the flower micelles described for HEUR polymers. 


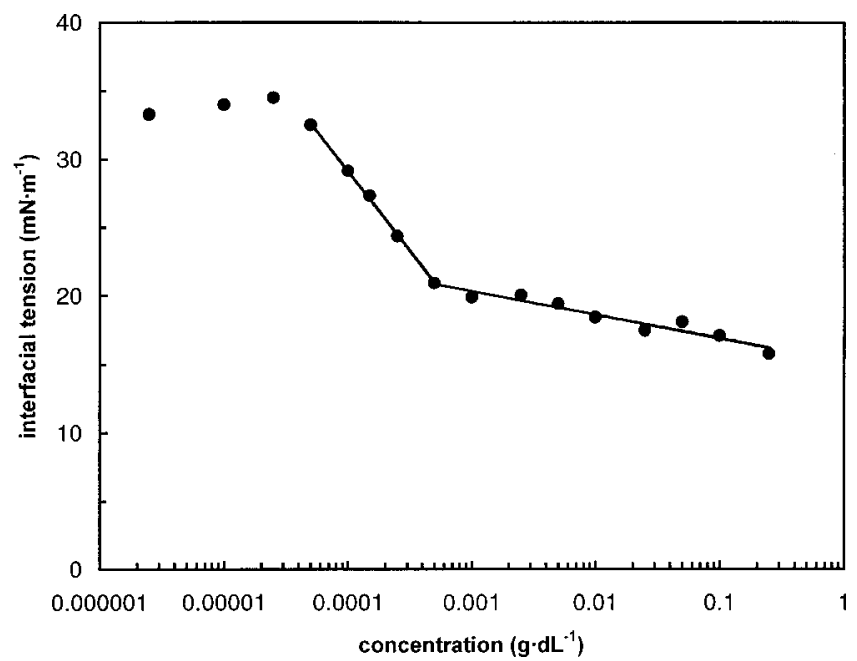

FIG. 1. Interfacial tension of HMHEC water solutions and toluene.

\section{B. Capillary viscometry}

Viscosity of dilute solutions of several HECs and of the HMHEC was measured in an Ubbelohde capillary viscometer. Results of reduced viscosity $\eta_{\text {red }}$ can be seen in Fig. 2. Intrinsic viscosity $[\eta]$ was also determined. The Huggins equation

$$
\eta_{\text {red }}=[\eta]+k^{\prime}[\eta]^{2} C_{p},
$$

where $C_{p}$ is the polymer concentration, was applied to the linear range, so that the Huggins coefficient $k^{\prime}$ could be calculated. The viscosity rise observed for HMHEC is attributed to intermicellar association. A recently published work [Reuvers (1999)] that studies HEURs relates this increase in the viscosity to the formation of micelles. From our interfacial measurements we can conclude that HMHEC micelles exist at much smaller concentrations than that at which the increase in viscosity is observed and, consequently, this increase in viscosity cannot be due to the formation of micelles. It has to be the consequence of interactions between the existing micelles. Moreover, we think that our results are in better agreement with the idea of loop-to-bridge transition, which argues that at low concentrations micelles are independent with loops predominating. A gradual loop-to-bridge transition occurs at increasing concentration, with an increasing number of bridges being present. The calculated values of $[\eta]$ and $k^{\prime}$ are listed in Table II. In general, $k^{\prime}$ is around $0.3-0.5$ for polymers in good solvents [Stickler and Sütterlin (1989)]. The higher value for the HMHEC is an indication of polymer associations. Intrinsic viscosity is a measure of the hydrodynamic volume of polymers in the limit of low concentrations. This dependence can be described by the Kuhn expression for a linear chain molecule [Ullmann's (1991)]

$$
[\eta]=K M^{\alpha} .
$$

The behavior of the four HECs studied can be fitted to this expression, with $\alpha$ $=0.80$. However, the data for HMHEC deviate from the fitting and has a lower value. The smaller $[\eta]$ can be related with the volume reduction produced by a more compact packing of the HMHEC molecules through intramolecular hydrophobic association [Sau and Landoll (1989)]. Although [ $\eta]$ is usually related to an individual molecule, this is presumably not the case for the HMHEC because aggregation has been found to exist at 

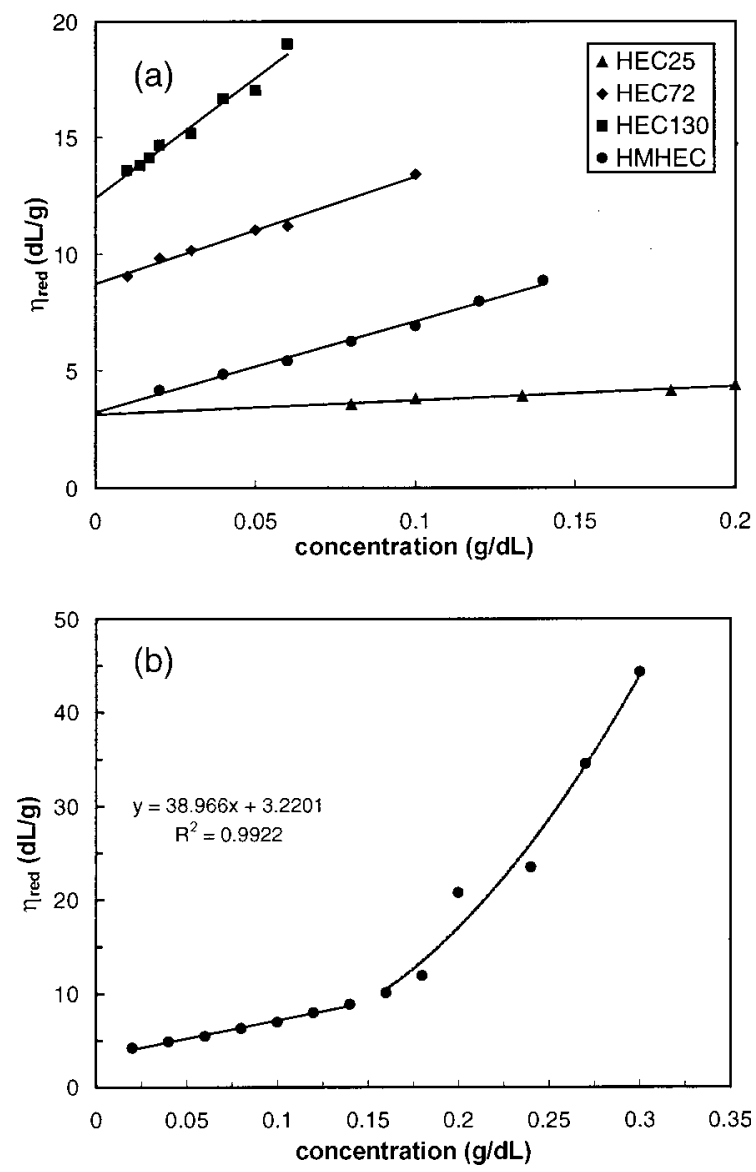

FIG. 2. (a) Reduced viscosity for several HEC and HMHEC. (b) Reduced viscosity for HMHEC.

much lower concentrations than that used to obtain reduced viscosity. So, as Nishikawa and co-workers (1998) argue, it is not a truly intrinsic viscosity but describes the properties of small aggregates consisting of several polymer molecules. Using fluorescence methods, these authors found that aggregation even occurs at concentrations as low as $0.001 \mathrm{~g} / \mathrm{dL}$ of HMHEC, which was the most diluted solution they studied. In the same way, Yekta et al. (1995) found HEUR associations to exist at concentrations as low as a few ppm. Nishikawa claims that HMHEC micelle-like aggregations are very small, because large steric requirements of the stiff cellulose backbone limit the number of hydro-

TABLE II. Viscometry data.

\begin{tabular}{lrc}
\hline \hline Polymer & {$[\eta]$} & $k^{\prime}$ \\
\hline HEC9 & 1.59 & 0.41 \\
HEC25 & 3.16 & 0.59 \\
HEC72 & 8.80 & 0.59 \\
HEC130 & 12.56 & 0.65 \\
HMHEC & 3.51 & 3.27 \\
\hline \hline
\end{tabular}




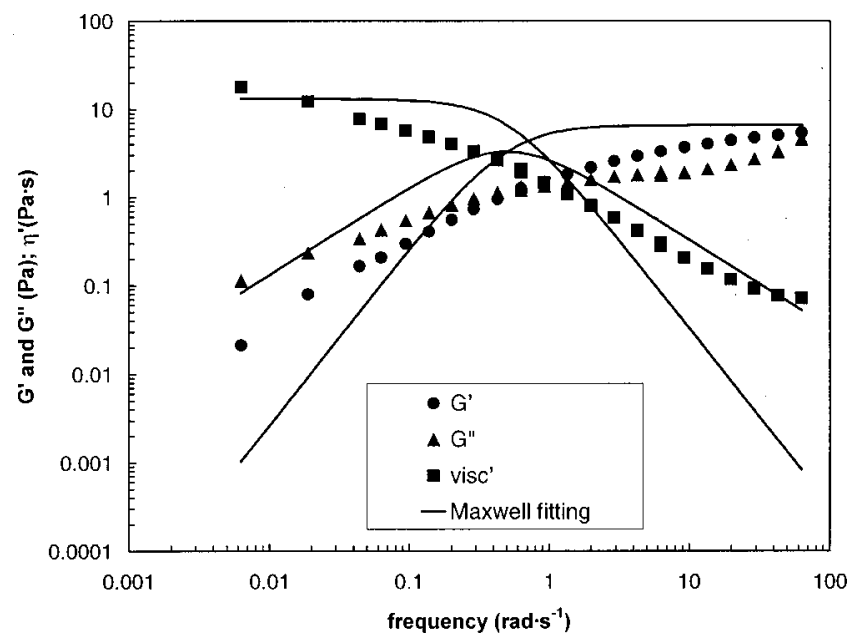

FIG. 3. Frequency sweep HMHEC $0.75 \% 20^{\circ} \mathrm{C}$. Points are experimental data, lines are the fitting for a Maxwell model with crossover as the relaxation time and $G^{\prime}$ at the higher frequency as $G_{\infty}$.

phobes that can come together. These conclusions are in agreement with our results: the cac we found is very small and the lack of stabilization of interfacial tension indicates small micelles.

In conclusion, HMHEC molecules are individually dissolved in water up to a few ppm, where small micelle-like aggregates are formed. A loop-to-bridge transition seems to exist when concentration is increased and network formation begins to take place. This is, qualitatively, the same behavior that Alami et al. (1996) observed when working with HEURs.

\section{Viscoelastic characterization}

Oscillatory measurements were carried out with solutions of HMHEC. Figure 3 shows how a single Maxwell model is not able to fit experimental data, and this is in agreement with previous works [Svanholm et al. (1997); Karlson et al. (2000)]. The smaller slope at low frequencies and its more gradual change around the crossover indicate a quite broad distribution of relaxation times, unlike what happens with linear telechelic HEURs. This distinct behavior must be related to the different architecture of the molecule. Svanholm attributed this behavior to the stiffness of the backbone because he observed that a distribution was required when working with HMHEC or aromatic HEURs, which are quite stiff. Aliphatic HEUR backbone consists of a polyethylene oxide (PEO) chain, which is very flexible. The location of hydrophobes is known in HEURs, because it depends on the position of urethane groups. There are two groups located at the ends of the linear chain in telechelic HEURs. Thus, when a hydrophobic end is taking part in a micelle, as can be seen in Fig. 4(a), and leaves it, the whole chain can relax because the bridge is broken. Therefore, chain relaxation time can be related to the inverse of the exit rate of the hydrophobic group from the micelle, as Annable and Buscall (1993) proposed in their theory. Relaxation time depends on the length of the hydrophobic group and is not much influenced by the backbone [Xu et al. (1996)]. This is thought to be due to the fact that PEO chains can easily adapt to all configurations. As hydrophobic groups and micelles are all the same, there is only a single exit rate and, as a result, a single relaxation time. On the other hand, these groups are randomly distributed along the 

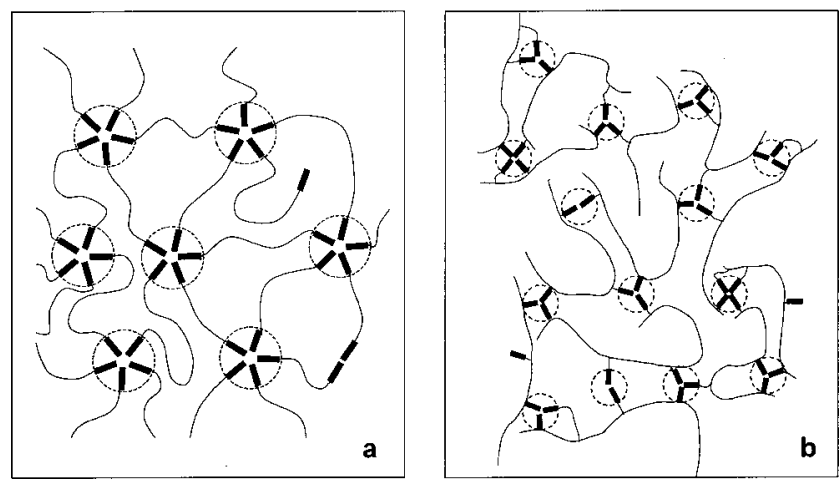

FIG. 4. Micellar network in solution (a) for a linear telechelic HEUR with strong hydrophobic groups, larger than $\mathrm{C} 12$; (b) for a HMHEC.

backbone of the HMHEC [Fig. 4(b)], since, when they are added to the HEC molecule, they can react with all $-\mathrm{OH}$ available, although for steric reasons they find it easier to react with $-\mathrm{OH}$ of oligomeric ethylene oxide chains [Zhang (2001)]. The cellulose backbone is quite stiff, so that some tension can be generated in a chain that pulls at hydrophobes in micelles and thus promotes their disengagement and affects relaxation time. The tension that a hydrophobe has to support depends on its position in the chain of cellulose. As a result, a distribution of relaxation times is observed. When the work of $\mathrm{Xu}$ et al. (1997) is studied, another reason for this behavior is found: they required a distribution of relaxation times to fit viscoelasticity, not of telechelic but of comb HEURs. Whereas for telechelic HEURs the disengagement of a hydrophobe necessarily implies relaxation of the whole chain and breaking of the elastically effective chain, in comb associative polymers, as can be seen in Fig. 4(b), the complete relaxation of the molecule needs the exit of more than one hydrophobe. As hydrophobes are randomly distributed along the backbone and may participate in different micelles, a distribution of relaxation times exists.

We do not believe that these two effects exposed by Svanholm and Xu are necessarily in conflict, and both can contribute to the presence of a distribution of relaxation times.

Figure 3 shows that the frequency-dependent loss modulus undergoes two increases. This implies two relaxation processes. The longer relaxation process is thought to correspond to the exit of hydrophobes from micelles, as stated earlier. The shorter relaxation time observed at high frequencies is attributed to rapid movements of free chains, and may follow the Rouse model or some other models that introduce stiffness of the molecule or hydrodynamic interactions, such as the Zimm theory [Ferry (1980)].

A logarithmic distribution of chain disengagement times around a main relaxation time, $\lambda_{M}$, is proposed, with a standard deviation, $\sigma$. So, if a generalized Maxwell model is applied, three parameters are required: $\lambda_{M}, \sigma$, and the plateau modulus, $G_{N}$, related to the number density of elastically active chains. Knowledge of the Rouse relaxation process is poor, due to experimental limitations, since it mostly occurs at times so short that they are outside the range of frequencies tested. Consequently, Rouse parameters cannot be known separately and they are reduced to a single parameter with viscosity units termed viscosity at infinite frequency, $\eta_{\infty}$. Therefore, oscillatory data can be fitted to the equations 

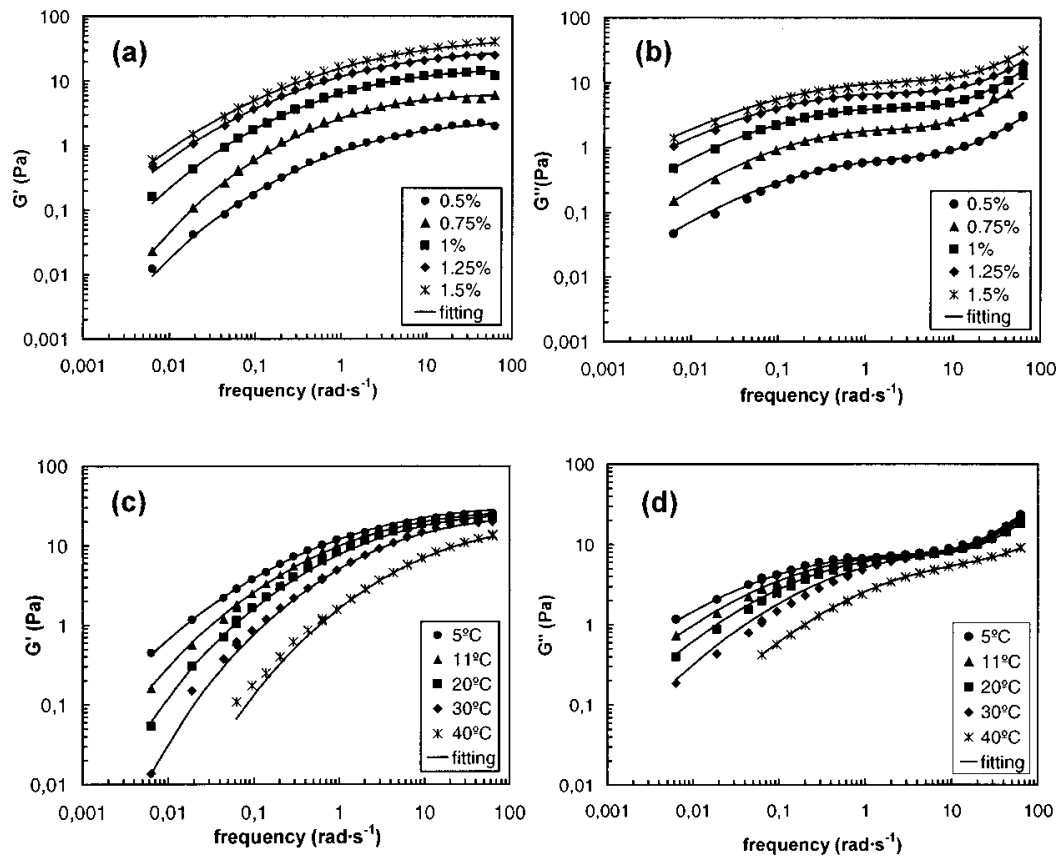

FIG. 5. Oscillatory data and fitting for HMHEC water solutions. (a) and (b) are $G^{\prime}$ and $G^{\prime \prime}$ at $5{ }^{\circ} \mathrm{C}$ and several concentrations. (c) and (d) are $G^{\prime}$ and $G^{\prime \prime}$ at a concentration of $1.25 \%$ and several temperatures.

$$
\begin{gathered}
G^{\prime}=\int_{-\infty}^{+\infty} H(\ln \lambda) \frac{(\omega \lambda)^{2}}{1+(\omega \lambda)^{2}} d \ln \lambda, \\
G^{\prime \prime}=\int_{-\infty}^{+\infty} H(\ln \lambda) \frac{\omega \lambda}{1+(\omega \lambda)^{2}} d \ln \lambda+\omega \eta_{\infty},
\end{gathered}
$$

where $H(\ln \lambda)$ is described by a logarithmic distribution according to

$$
H(\ln \lambda)=\frac{G_{N}}{\ln \sigma \sqrt{2 \pi}} \exp \left[-\frac{\left(\ln \lambda-\ln \lambda_{M}\right)^{2}}{2(\ln \sigma)^{2}}\right] .
$$

The model is capable of fitting the experimental data very well, as can be seen in Fig. 5. It uses four parameters that seem to have physical significance. The study of the evolution of these parameters with some variables, such as temperature and concentration, will be useful to understand the thickening mechanism.

\section{Dependence of $G_{N}$ on temperature and concentration}

The dependence of $G_{N}$ on temperature and concentration can be appreciated from the data in Fig. 5. Figures 5(a) and 5(b) show that when concentration is increased, curves are shifted to higher values of storage and loss modulus, $G^{\prime}$ and $G^{\prime \prime}$, the shape being maintained. It indicates that the plateau modulus $G_{N}$ rises when concentration is increased. Figure 6 shows logarithmic distributions calculated for these curves through Eq. (6). These curves include only relaxation due to the exit of hydrophobes from micelles. They do not include Rouse relaxation because Rouse parameters cannot be known separately. In fact, global relaxation spectra would be the sum of both contributions. The 


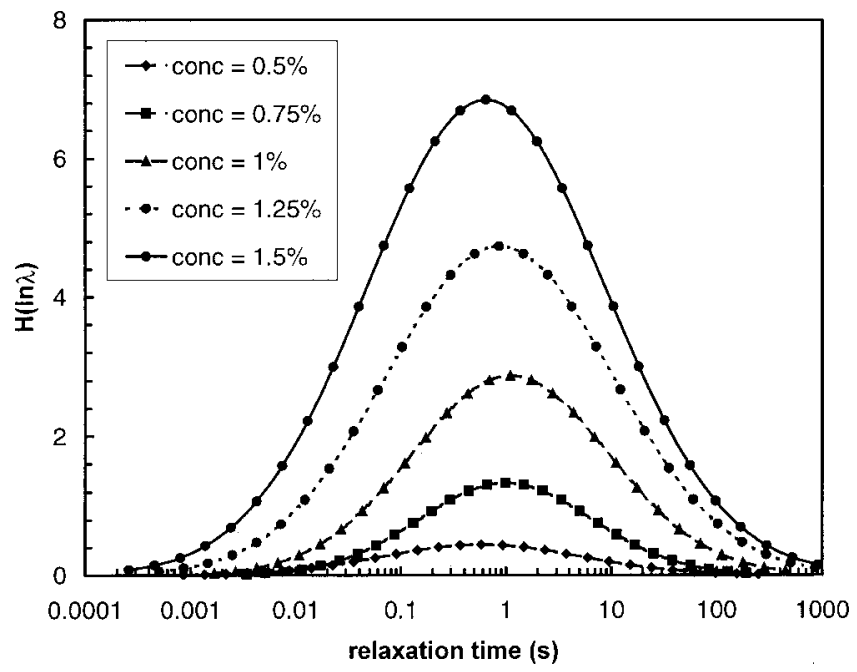

FIG. 6. Logarithmic distribution of relaxation times obtained for fittings of Figs. 5(a) and 5(b).

figure shows the complete logarithmic distributions, which cover seven orders of magnitude. But, of course, they are only meaningful in the range of frequencies studied and, as will be shown later, they can be extended to the range covered by the master curve. The plateau modulus $G_{N}$, which can be identified with the area below the curves, is seen to increase strongly with concentration. On the other hand, Figs. 5(c) and 5(d) show that when temperature is raised the curves move towards higher frequencies, but virtually maintaining their value on the $y$ axis, so that $G_{N}$ seems not to be very much influenced by $T$, except at $40^{\circ} \mathrm{C}$, where lower values are always obtained. Some change in structure produced around this temperature thus seems apparent. Nevertheless, at this temperature experimental reproducibility is poor, probably due to the low value observed for viscosity, which prevents it from remaining in the linear range because deformation is too great-even though experimentally applied shear stresses are as small as possible. Moreover, fitting obtained at $40^{\circ} \mathrm{C}$ is less satisfactory than for the other temperatures, probably because these experiments were not carried out in the linear range. The lack of influence of $T$ on $G_{N}$ can be appreciated when values obtained for $G_{N}$ are plotted against temperature (Fig. 7). Solid lines represent mean values.

The plateau modulus, $G_{N}$, could be related to the number density of elastically active chains, $\nu$, according to the equation [Annable et al. (1994)]

$$
G_{N}=\nu k T,
$$

as Green and Tobolsky (1946) predict in their transient network theory, based on rubber elasticity. Elastically active chains are related to bridges between micelles [Annable et al. (1996)]. When temperature increases, $\nu$ decreases due to Brownian motion, and this effect cancels - in fact sometimes very slightly overcomes - the direct effect of temperature on $G_{N}$ as can be deduced from Eq. (7) [Larson (1999)].

Master curves were built through results obtained at different temperatures. As $T$ does not influence $G_{N}$, scaling has to be performed only for frequency, and not for $G^{\prime}(\omega)$ and $G^{\prime \prime}(\omega)$, except $G^{\prime \prime}(\omega)$ at high frequencies, which should also be scaled in order to obtain a good superposition because $\eta_{\infty}$ changes with temperature. So, the contribution of $\eta_{\infty}(T)$ to $G^{\prime \prime}(\omega)$ was subtracted from results at experimental temperature $T$, and $\eta_{\infty}\left(T_{0}\right)$ 


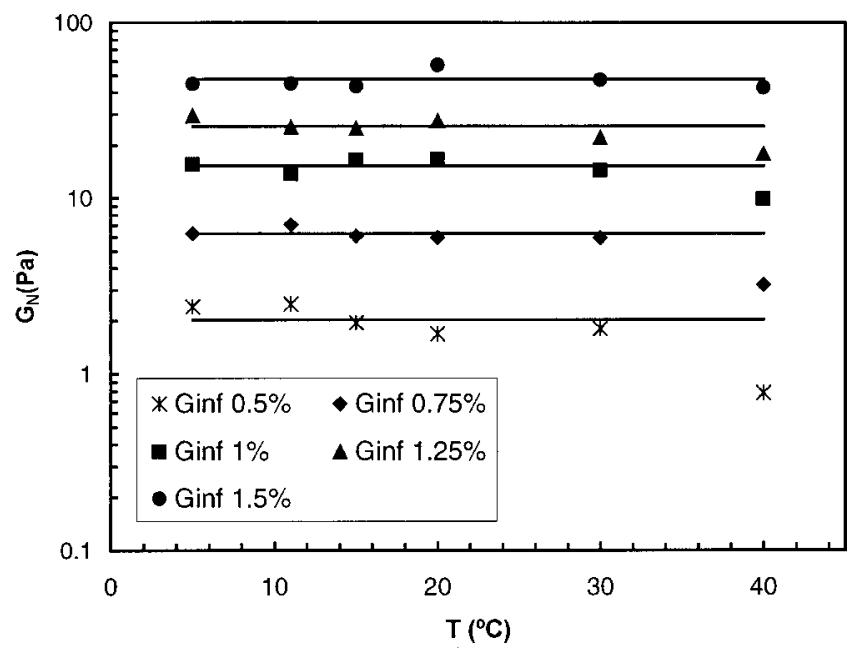

FIG. 7. $G_{N}$ vs $T$ at several concentrations of HMHEC. Continuous lines are the mean values.

calculated for experiments developed at the reference temperature, $T_{0}$, was added, multiplied by the reduced frequency $a_{T} \omega, a_{T}$ being the shifting factor for frequency. As a result, frequency was shifted as $\omega_{t \text { ref }}=a_{T} \omega_{\text {texp }}$, and $G^{\prime \prime}(\omega)$ was scaled as follows:

$$
G^{\prime \prime}(\omega)_{T_{0}}=G^{\prime \prime}(\omega)_{T_{\exp }}-\omega \eta_{\infty T_{\exp }}+a_{T} \omega \eta_{\infty T_{0}}
$$

where $T_{\exp }$ and $T_{0}$ are experimental and reference temperature, respectively. An example of master curves obtained in this way is given in Fig. 8. A reference temperature $T_{0}$ $=20^{\circ} \mathrm{C}$ was chosen. The fitting obtained for experimental data at $20^{\circ} \mathrm{C}$ is also plotted. It can be seen that the master curve obtained is reasonably good and is very well fitted for the model applied at $20^{\circ} \mathrm{C}$. Similar results are obtained when other concentrations are

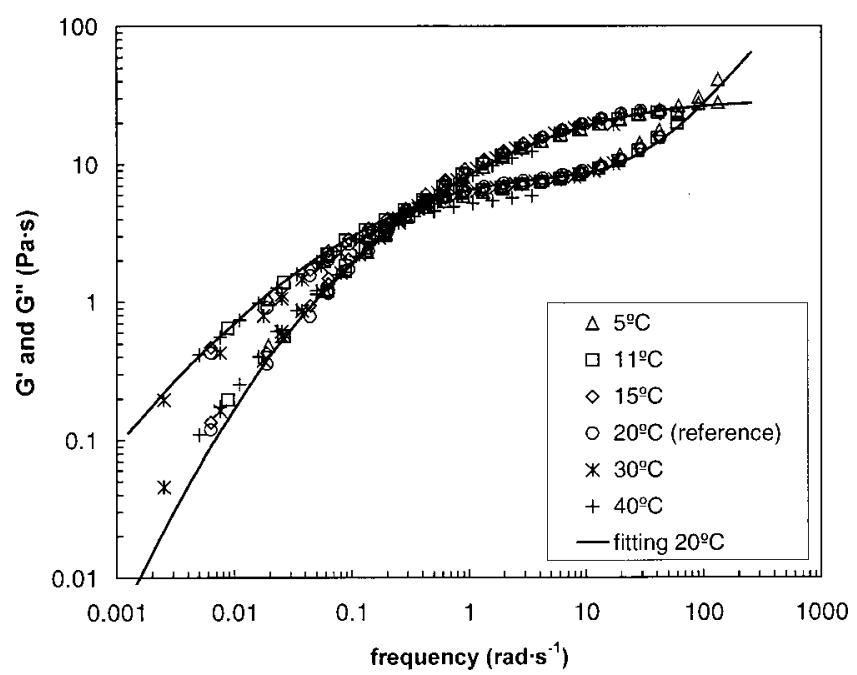

FIG. 8. Master curve at a reference temperature $T_{\text {ref }}=20^{\circ} \mathrm{C}$. Lines are the fitting at this temperature. Concentration is $1.25 \%$. 


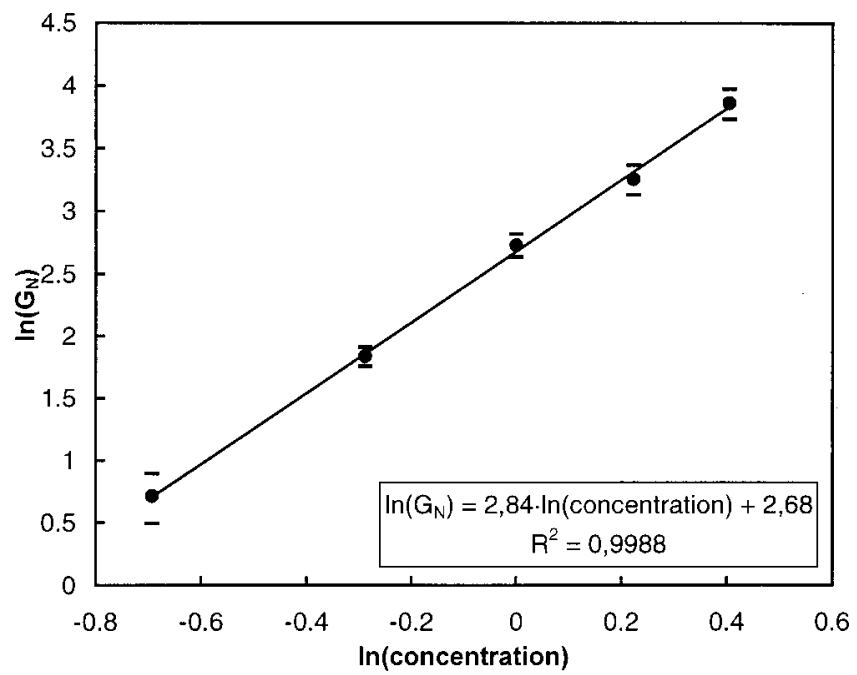

FIG. 9. $G_{N}$ mean vs concentration $\log -\log$ plot. Line corresponds to a linear regression whose expression is included in the graph area. Confidence intervals are also represented.

employed. This supports the idea that the model proposed seems adequate for describing rheological behavior of HMHEC aqueous solutions.

If independence of $T$ is assumed for $G_{N}$, a single mean value $G_{N M}$ can be obtained at each concentration, whatever temperature is used, and a standard deviation can be found for this new mean value. Dependence of $G_{N M}$ on concentration appears as a straight line if plotted in a double logarithmic way (Fig. 9). Mean confidence interval is also plotted. A dependence on concentration raised to 2.8 is observed. Tanaka and Edwards (1992) apply Eq. (7) to their polymeric systems. They only consider two possible situations for polymer chains: bridging chains, which are elastically effective, and dangling ends, which do not form part of the network. When the junction energy $E_{m}$ is large enough, the number of dangling ends can be neglected and nearly all chains are expected to be elastically active. As a result, the theory predicts a linear dependence of $G_{N}$ on concentration. Annable and Buscall (1993) find a linear dependence of $G_{N}$ on concentration for HEURs at high concentrations, but a quadratic or higher dependence at low concentrations. Like Yekta et al. (1993), they propose that this stronger dependence is due to the fact that at low concentrations not all the chains are elastically active. Instead, in most chains both sides are joined to the same micelle and form loops. These change into bridges between micelles and become elastically active when concentration is increased because micelles are closer together and interactions between them are easier. We relate the parameter $G_{N}$ with that used by Annable and calculated through Eq. (7). We find $G_{N}$ to depend on concentration raised to 2.8. So, dependence of $G_{N}$ on concentration is not linear but stronger. Our results are thus in agreement with those reported by Annable. Loop-to-bridge transitions are then accepted as taking place in HMHEC solutions.

\section{E. Dependence of $\lambda_{M}$ on temperature and concentration}

Figure 6 shows that the mean value of the distribution, corresponding to $\lambda_{M}$, seems not to be influenced by concentration. It was also seen for all temperatures tested, except for $40^{\circ} \mathrm{C}$, where $\lambda_{M}$ seems to decrease when concentration is increased. Problems of 


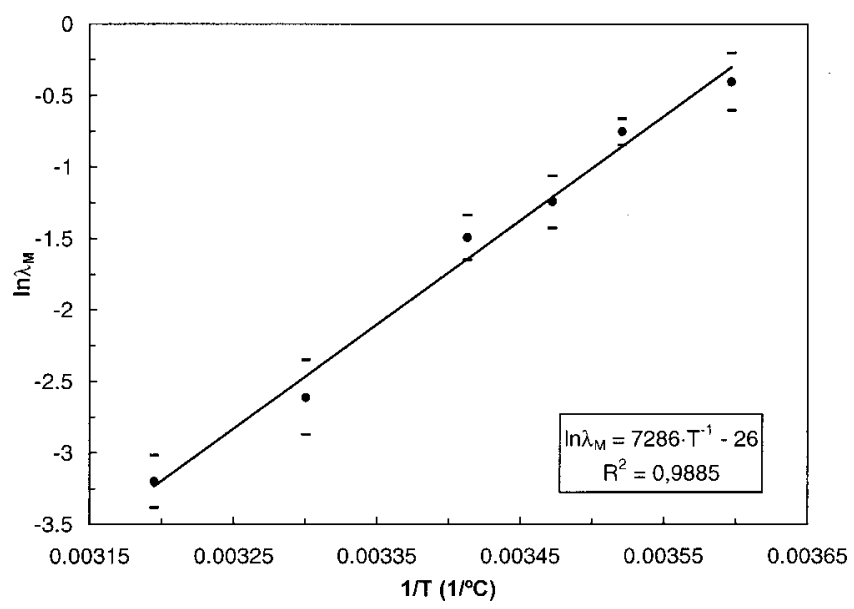

FIG. 10. Arrhenius plot of $\lambda_{M M}$ against temperature for HMHEC aqueous solutions.

experiments at this temperature have already been mentioned. Although the dependence of $\lambda_{M}$ on concentration cannot be completely ruled out through these results, they seem to indicate that mean relaxation time does not increase with concentration, unlike what happens with HEURs, and that no clear tendency is observed. Thus, in a first approximation it will be assumed that $\lambda_{M}$ does not change with concentration. Reptation theory, according to the Doi-Edwards model (1986), predicts that relaxation time, $\lambda$, depends on concentration raised to $3 / 2$, thus, reptation can be rejected for HMHEC. Annable and Buscall (1993) also find an increase in $\lambda$ with concentration. As stated earlier, these authors identify the inverse of relaxation time with the exit rate of a hydrophobe from its micelle. Through fluorescence experiments, Tam et al. (1998) prove that HEUR micellar size does not change in a wide range of concentrations for strong hydrophobic groups. This indicates that the activation energy of the junction of a hydrophobe to its micelle $E_{m}$ and, as a result, the relaxation time related to it, should be constant. Consequently, the dependence of $\lambda$ on concentration observed for HEURs must be assigned to other causes. It has been related to the presence of superbridges in the micellar network at low concentrations, formed by the junction of several micelles with a functionality of 2 , so that each micelle is connected to two neighboring micelles and a linear structure is formed. An elastically effective chain is destroyed when one of the junctions between micelles along the chain is broken. The longer the superchain, the more junctions it has and, as a consequence, the probability of one of them being broken is greater. As a result, the relaxation time decreases when superchains are present. When concentration is increased, there is a transition from intramicellar loop associations to intermicellar bridges, functionality of micelles increases and superchains become shortened by new associations.

On the other hand, the presence of superchains seems not to be important in HMHEC solutions, because $\lambda_{M}$ is not shorter at lower concentrations. This may be due to the fact that linear superchains practically do not exist in HMHEC solutions, since comb structure prevents the formation of linear structures and favors branched ones. As each molecule of thickener has several hydrophobes, it can be joined at all those points and tends to form a more ramified network, with few superchains.

If $\lambda_{M}$ is assumed to be independent of concentration for HMHEC, a mean value $\lambda_{M M}$ that includes $\lambda_{M}$ at all concentrations can be calculated for each temperature. Results are plotted in Fig. 10 through an Arrhenius representation. Short lines indicate a probability 


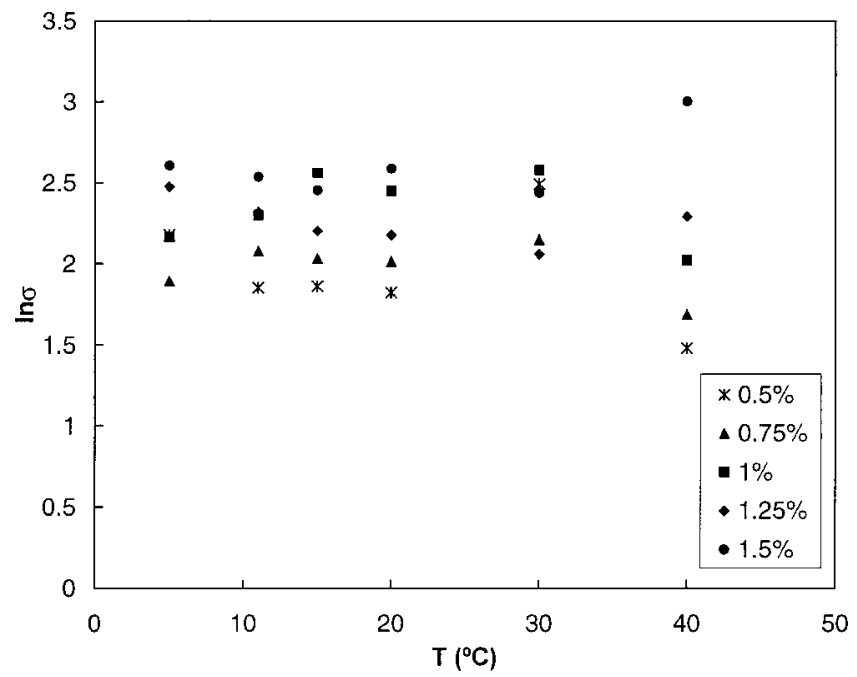

FIG. 11. $\ln \sigma$ vs temperature for several concentrations of HMHEC.

confidence interval of $95 \%$.

Points represented in this way fit a straight line that is always inside the confidence intervals, indicating that the dependence on temperature of $\lambda_{M M}$ follows an Arrhenius law like Eq. (1), as does HEUR relaxation time [Annable et al. (1994); Tam et al. (1998)]. $E_{m}$ is estimated to be around $60 \mathrm{~kJ} \mathrm{~mol}^{-1}$. Annable finds $E_{m} \simeq 70 \mathrm{~kJ} \mathrm{~mol}^{-1}$ for the HEUR used in his experiments. It can be seen that both are of the same order. The good Arrhenius law fit, without tendencies, and the likeness of both $E_{m}$ obtained for HEUR and HMHEC are further indications that linear viscoelasticity of HMHEC can be described by a generalized Maxwell model where $H(\ln \lambda)$ has the form of a logarithmic distribution.

\section{F. Dependence of In $\sigma$ on temperature and concentration}

The dependence of the fitting parameter $\ln \sigma$ on temperature and concentration was studied and $\ln \sigma$ values are plotted against temperature in Fig. 11. $\ln \sigma$ does not seem to be influenced by temperature. All results are around 2.2, and tend to increase with concentration. This indicates a broad relaxation spectrum that widens slightly with concentration and, as a consequence, with the quantity of micelles present in the medium. HMHEC structure might explain these results. As a consequence of the comb structure of HMHEC, although all hydrophobes can take part in the micellar network, some are more likely to form part of the network than others, depending on the distance to the ends of the chain and on neighboring hydrophobic groups. Kaczmarski and Glass (1993) studied HEUR solutions with different molecular weights, i.e., with different distances between hydrophobes, concluding that low molecular weight HEURs with short distances between hydrophobes tend to form intramicellar junctions. On the other hand, HEURs that have hydrophobes separated by long chains are more liable to form bridges at low concentrations. Whether a similar behavior is accepted for HMHEC, some hydrophobes seem to be situated in favorable positions and form the network at the lower concentrations. In this case, dispersion of relaxation times is relatively small. When concentration is increased, there are more micelles in the medium and they are closer together. Thus, the probability of hydrophobes located in less favored positions forming part of the network is larger. 


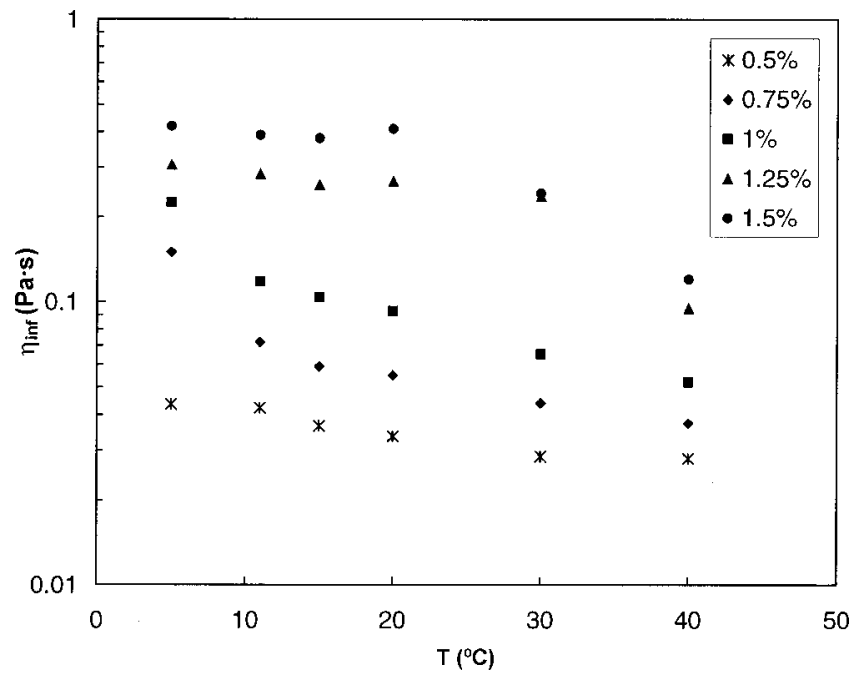

FIG. 12. $\eta_{\infty}$ vs temperature at different concentrations of HMHEC.

The major variety of junctions that forms elastically effective chains produces a wider relaxation time distribution; therefore, $\ln \sigma$ increases.

\section{G. Dependence of $\boldsymbol{\eta}_{\infty}$ on temperature and concentration}

In order to analyze $\eta_{\infty}$ dependence on temperature and concentration, Fig. 12 is presented. Viscosity at high frequency, $\eta_{\infty}$, decreases with temperature and increases with concentration. This parameter is related to a rapid Rouse-like relaxation process. Rouse parameters cannot be known separately, due to experimental limitations. When temperature is increased, Brownian motion facilitates relaxation processes. As a result, $\eta_{\infty}$ decreases. When concentration and, thus, number of chains present in the medium is increased, relaxation of chains is more difficult and $\eta_{\infty}$ increases.

\section{CONCLUSIONS}

Interfacial tension and capillary viscometry measurements indicate that HMHEC is molecularly dissolved up to a few ppm. At higher concentrations, molecules associate in aqueous solution through their hydrophobic groups. They then form independent micelles that can freely move across the medium, and when concentration is increased micelles gradually begin to interact and associate. As a consequence, a three-dimensional network begins to extend which highly increases system viscosity.

Oscillatory results obtained with solutions of HMHEC cannot be fitted if a singleelement Maxwell model is used, unlike what happens with telechelic HEURs, and a spectrum of relaxation times is required. Differences between these two groups of associative thickeners are attributed to the stiffness and comb structure of the HMHEC.

Rheology of HMHEC aqueous solutions can be fitted to a generalized Maxwell model that assumes a relaxation spectrum with the shape of a logarithmic distribution related to chain disengagements plus a parameter related to individual chain Rouse-like relaxation. Thus, four parameters are required: a mean relaxation time, $\lambda_{M}$; a standard deviation around this time, $\sigma$; a plateau modulus, $G_{N}$; and a viscosity at high frequency, $\eta_{\infty}$. 
$G_{N}$ increases with concentration due to loop-to-bridge transitions, as happens with HEURs, and it is not modified by temperature, due to the fact that the direct effect of $T$ is masked by the diminution of the number of elastically active chains.

Like HEUR single relaxation time, $\lambda_{M}$ has an Arrhenius dependence on temperature. The lack of influence of concentration on $\lambda_{M}$ seems to be related to the fact that the comb structure of HMHEC prevents the presence of superbridges.

\section{References}

Alami, E., M. Rawiso, G. Beinert, W. Binana-Limbele, and J. François, "Model hydrophobically end-capped poly(ethylene oxide) in water," Adv. Chem. Ser. 248, 343-361 (1996).

Annable, T. and R. Buscall, "The rheology of solutions of associating polymers: Comparison of experimental behavior with transient network theory," J. Rheol. 37, 695-726 (1993).

Annable, T., R. Buscall, and R. Ettelaie, "Network formation and its consequences for the physical behavior of associating polymers in solution," Colloids Surf., A 112, 97-116 (1996).

Annable, T., R. Buscall, R. Ettelaie, P. Shepherd, and D. Whittlestone, "Influence of surfactants on the rheology of associating polymers in solution," Langmuir 10, 1060-1070 (1994).

Brown, R., "Advances on thickener technology for waterborne coatings," Eur. Polym. J. 184, 267-270 (1994).

Doi, M. and S. F. Edwards, The Theory of Polymer Dynamics (Clarendon, Oxford, 1986).

Ferry, J. D., Viscoelastic Properties of Polymers, 3rd ed. (Wiley, New York, 1980).

Glass, J. E., D. N. Schulz, and C. F. Zukoski, "Polymers as rheology modifiers," ACS Symp. Ser. 462, 2-17 (1991).

Goodwin, J. W., R. W. Hughes, C. K. Lam, J. A. Miles, and B. C. H. Warren, "The rheological properties of a hydrophobically modified cellulose," Adv. Chem. Ser. 223, 365-378 (1989).

Green, M. S. and A. V. Tobolsky, "A new approach to the theory of relaxing polymeric media," J. Chem. Phys. 14, 80-89 (1946).

Jenkins, R. D., C. A. Silebi, and M. S. El-Aasser, "Steady-shear and linear-viscoelastic material properties of model associative polymer solutions," ACS Symp. Ser. 462, 222-233 (1991).

Kaczmarski, J. P. and J. E. Glass, "Synthesis and solution properties of hydrophobically-modified ethoxylated urethanes with variable oxyethylene spacer lengths," Macromolecules 26, 5149-5156 (1993).

Karlson, L., F. Joabson, and K. Thuresson, "Phase behavior and rheology in water and in model paint formulations thickened with HM-EHEC: Influence of the chemical structure and the distribution of hydrophobic tails," Carbohydr. Polym. 41, 25-35 (2000).

Karunasena, A., G. Brown, and J. E. Glass, "Hydrophobically modified ethoxylated urethane architecture. Importance for aqueous- and dispersed-phase properties," Adv. Chem. Ser. 223, 495-525 (1989).

Kroon, G., "Associative behavior of hydrophobically modified hydroxyethyl celluloses (HMHECs) in waterborne coatings," Prog. Org. Coat. 22, 245-260 (1993).

Landoll, L. M., "Nonionic polymer surfactants," J. Polym. Sci. 20, 443-455 (1982).

Larson, R. G., The Structure and Rheology of Complex Fluids (Oxford University Press, New York, 1999).

Nishikawa, K., A. Yekta, H. H. Pham, M. A. Winnik, and A. C. Sau, "Fluorescence studies of hydrophobically modified hydroxyethylcellulose (HMHEC) and pyrene-labeled HMHEC," Langmuir 14, 7119-7129 (1998).

Piculell, L., F. Guillemet, K. Thuresson, V. Shubin, and O. Ericsson, "Binding of surfactants to hydrophobically modified polymers," Adv. Colloid Interface Sci. 63, 1-21 (1996).

Reuvers, A. J., "Control of rheology of water-borne paints using associative thickeners," Prog. Org. Coat. 35, 171-181 (1999).

Sau, A. C. and L. M. Landoll, "Synthesis and solution properties of hydrophobically modified (hydroxyethylcellulose," Adv. Chem. Ser. 223, 343-363 (1989).

Semenov, A. N., J. F. Joanny, and A. R. Kmokhlov, "Associating polymers: Equilibrium and linear viscoelasticity," Macromolecules 28, 1066-1072 (1995).

Sivadasan, K. and P. Somasundaran, "Polymer-surfactant interactions and the association behavior of hydrophobically modified hydroxyethylcellulose," Colloids Surf., A 49, 229-239 (1990).

Stickler, M. and N. Sütterlin, in Polymer Handbook, 3rd ed., edited by J. Brandrup and H. Immergut (Wiley, New York, 1989).

Svanholm, T., F. Molenaar, and A. Toussaint, “Associative thickeners: their adsorption behavior onto latexes and the rheology of their solutions," Prog. Org. Coat. 30, 159-165 (1997).

Tam, K. C., R. D. Jenkins, M. A. Winnik, and D. R. Basset, "A structural model of hydrophobically modified urethane-ethoxylated (HEUR) associative polymers in shear flow," Macromolecules 31, 4149-4159 (1998).

Tanaka, F. and S. F. Edwards, "Viscoelastic properties of physically cross-linked networks. Transient network theory," Macromolecules 25, 1516-1523 (1992). 
Tanaka, R., J. Meadows, P. A. Williams, and G. O. Phillips, "Interactions of hydrophobically modified (hydroxyethyl)cellulose with various added surfactants," Macromolecules 25, 1304-1310 (1992).

Ullmann's Encyclopedia of Industrial Chemistry VCH, 5th ed. (1991), Vol. A18, pp. 400-436.

Wang, Y. and M. A. Winnik, "Onset of aggregation for water-soluble polymeric associative thickeners-A fluorescence study," Langmuir 6, 1437-1439 (1990).

Wetzel, W. H., M. Chen, and J. E. Glass, "Associative thickeners. An overview with an emphasis on synthetic procedures," Adv. Chem. Ser. 248, 163-179 (1996).

Xu, B., A. Yekta, Z. Masoumi, and M. A. Winnik, "The functionality of associative polymer networks: The association behavior of hydrophobically modified urethane-ethoxylated (HEUR) associative polymers in aqueous solution," Colloids Surf., A 112, 239-250 (1996).

$\mathrm{Xu}, \mathrm{B}$., A. Yekta, and M. A. Winnik, "Viscoelastic properties in water of comb associative polymers based on poly(ethylene oxide)," Langmuir 13, 6903-6911 (1997).

Yekta, A., J. Duhamel, H. Adiwidjaja, P. Brochard, and M. A. Winnik, "Fluorescence studies of associating polymers in water: Determination of the chain end aggregation number and a model for the association process," Macromolecules 28, 956-966 (1995).

Yekta, A., B. Xu, J. Duhamel, H. Adiwidjaja, and M. A. Winnik, “Association structure of telechelic associative thickeners in water," Langmuir 9, 881-883 (1993).

Zhang, L. M., "Cellulosic associative thickeners," Carbohydr. Polym. 45, 1-10 (2001). 